\title{
Women with epilepsy
}

In this issue of Functional Neurology, Bangar et al. address the main issues of practical relevance to clinicians treating female patients with epilepsy: epidemiology and clinical presentations (including catamenial epilepsy), contraception, reproductive and sexual dysfunction, pregnancy, lactation, menopause-related issues (including bone health), and mental health aspects.

As mentioned by Bangar et al., safety data on newer anti-seizure medications are sparse. Also there is a lack of objective information about how the risks of behavioral and cognitive problems in offspring differ with amounts of medication exposure to the fetus. Levels of anti-seizure medication in the umbilical cord and breast milk are not known.

Hormonal fluctuations in women with epilepsy have a direct impact on their seizure thresholds. Progesterone and estrogen act as neurosteroids in the central nervous system; they have short-term effects on neuronal membrane stabilization and long-term genomic effects, with estrogen being excitatory and progesterone inhibitory (Harden et al., 2013). In women, seizures are frequently catamenial, occurring primarily during specific phases of the menstrual cycle. Some studies suggest that catamenial seizures occur in up to $75 \%$ of all women with epilepsy. However, when defined as a consistent doubling of seizure frequency, catamenial epilepsy is seen in one third of women, mostly with focal epilepsies (Herzog et al., 1997). Moreover, pregnancy is associated with unique hormonal surges and metabolic changes that influence seizure risk and serum levels of anti-seizure medications.

Despite the well-studied role of sex hormones in epilepsy, the only double-blinded placebo-controlled trial of progesterone in catamenial epilepsy failed to show superiority of progesterone over placebo. However in a secondary analysis, a subset of women with more than threefold catamenial exacerbation of seizure frequency were responsive to progesterone treatment (Herzog et al., 2012). Apart from progesterone, strategies to tackle catamenial seizures include the use of acetazolamide, clobazam, and increasing the dose of baseline anti-seizure medication during the anticipated seizure exacerbation phase.

There are known interactions between anti-seizure medications and sex hormones. Enzyme inducers can decrease the concentration of sex hormones, and hormonal contraceptives may increase the elimination of certain anticonvulsants such as lamotrigine. There is a high risk of contraceptive failure when enzyme-inducing antiseizure medications are used with hormonal contraceptives. Barrier methods of contraception are thus useful in women with epilepsy. The most reliable form of reversible contraception for any woman is the intrauterine device, and this is considered the contraceptive method of choice for most women with epilepsy (Gerard and Meador, 2016).

Management of epilepsy during pregnancy poses a special challenge because of the need to balance teratogenic effects of anti-seizure medications with maintenance of seizure control. Ongoing seizures during pregnancy can cause complications ranging from fetal bradycardia, fetuses that are small for gestational age, and possibly still births. Women with epilepsy seeking pregnancy have comparable chances of achieving pregnancy, time to achieve pregnancy, and pregnancy outcomes, compared with healthy peers (French et al., 2016). Epilepsy is one of the most common chronic neurological disorders requiring continuous treatment throughout pregnancy. Seizure frequency remains unchanged during the majority of pregnancies (EURAP Study Group, 2002).

Anti-seizure medications can increase the risk of major congenital malformations (MCMs) by up to 3 times as compared to the general population. Lamotrigine and levetiracetam are the safest anti-seizure medications with regard to teratogenicity and cognitive outcome of the child (Pennell, 2008). Valproic acid deserves a special mention, as the risk of MCMs with valproic acid monotherapy in the first trimester can be 6 to $9 \%$ (Hernández-Díaz et al., 2012; Tomson et al., 2011). This treatment also negatively impacts on the neurodevelopmental outcomes of children born to women with epilepsy, being associated with reduced cognitive abilities across a range of domains, including low IQ (Meador, 2002). In general, anti-epileptic polytherapy should be avoided in pregnancy, especially when valproic acid is a part of the regimen (Harden et al., 2009a,b).

As mentioned, women with epilepsy are as likely to achieve pregnancy as their healthy peers and will have similar pregnancy outcomes. More data are needed regarding pregnancy-related complications, neonatal outcomes and C-section rates, incidence of postpartum depression and anxiety in women with epilepsy as compared to the 
general population. The Maternal and Neurodevelopmental Outcomes of in Utero Antiepileptic Drug (AED) Exposure (MONEAD) study is a currently ongoing multicenter, prospective study. It is co-sponsored by Emory University, the EMMES Corporation and the NINDS. Overall goals of this study are to establish the relationship between antiepileptic drug exposure and outcomes in mother and child and to describe and explain the variability in antiepileptic drug exposure and response. The study may well answer some of the questions concerning various aspects of epilepsy in women and increase our knowledge on multiple levels to improve care and reduce adverse outcomes in women with epilepsy and their children.

Anumeha Sharma, MD

François Boller, MD, PhD

Mohamad Koubeissi, MD

Department of Neurology, George Washington University Medical School

Washington DC, USA

E-mail: anusharma@mfa.gwu.edu

\section{References}

Bangar S, Shastri A, Cavanna AE (2016). Women with epilepsy: clinically relevant issues. Funct Neurol 31 : $127-$ 134

EURAP Study Group (2006). Seizure control and treatment in pregnancy: observations from the EURAP epilepsy pregnancy registry. Neurology 66:354-360.

French J, Harden C, Pennell P, et al (2016). A prospective study of pregnancy in women with epilepsy seeking conception (The WEPOD Study). Neurology 86 Supplement S22.006 http://www.neurology.org/content/86/16_Supplement/S22.006.short

Gerard EE, Meador KJ (2016). Managing epilepsy in women. Continuum (Minneap Minn) 22:204-226.

Harden CL, Pennell PB (2013). Neuroendocrine considerations in the treatment of men and women with epilepsy. Lancet Neurol12:72-83.

Harden CL, Pennell PB, Koppel BS, et al (2009a). Practice parameter update: management issues for women with epilepsy, focus on pregnancy (an evidence-based review): vitamin $\mathrm{K}$, folic acid, blood levels, and breastfeeding: report of the Quality Standards Subcommittee and Therapeutics and Technology Assessment Subcommittee of the American Academy of Neurology and American Epilepsy Society. Neurology 73:142-149

Harden CL, Meador KJ, Pennell PB, et al (2009b). Practice parameter update: management issues for women with epilepsy: focus on pregnancy (an evidence-based review): teratogenesis and perinatal outcomes: report of the Quality Standards Subcommittee and Therapeutics and Technology Assessment Subcommittee of the American Academy of Neurology and American Epilepsy Society. Neurology 73:133-141.

Hernández-Díaz S, Smith CR, Shen A, et al (2012). Comparative safety of antiepileptic drugs during pregnancy. Neurology 78:1692-699.

Herzog AG, Klein P, Ransil BJ (1997). Three patterns of catamenial epilepsy. Epilepsia 38:1082-1088

Herzog AG, Fowler KM, Smithson SD, et al (2012). Progesterone vs placebo therapy for women with epilepsy: a randomized clinical trial. Neurology 78:1959-1966.

Meador KJ (2002). Neurodevelopmental effects of antiepileptic drugs. Curr Neurol Neurosci Rep 2: $373-378$.

Pennell PB (2008). Antiepileptic drugs during pregnancy: what is known and which AEDs seem to be safest? Epilepsia 49:43-55.

Tomson T, Battino D, Bonizzoni E, et al (2011). Dose-dependent risk of malformations with antiepileptic drugs: an analysis of data from the EURAP epilepsy and pregnancy registry. Lancet Neurol 10: 609-617. 